ERRATUM

\title{
Ranbaxy loses in Caduet dispute
}

Charlotte Harrison

Nature Reviews Drug Discovery 7, 113 (February 2008) | doi:10.1038/nrd2516

This article stated that Caduet is covered by three US patents US 4,681,893 (which covers atorvastatin calcium); 5,273,995 (which covers amlodipine besylate); and 6,455,574 (which covers the product). In fact, both the ' 893 and the ' 995 patents relate to atorvastatin calcium. 